\title{
Should eponyms be abandoned?
}

Alexander Woywodt consultant renal physician, Renal Unit, Lancashire Teaching Hospitals NHS Trust, Preston, Lancashire PR2 9HT Eric Matteson professor of rheumatology, Mayo Clinic College of Medicine, Rochester, Minnesota, USA

Correspondence to: A Woywodt

Mail@Alexander-Woywodt.com

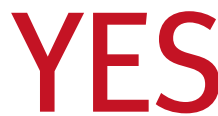

The Oxford English Dictionary defines an eponym as a person ... after whom a discovery, invention, institution, etc is named or thought to be named. Eponyms are deeply rooted in tradition and their use has long been viewed as a matter of taste. However, it is time to abandon them in favour of a more descriptive nomenclature.

Eponyms often provide a less than truthful account of how diseases were discovered and reflect influence, politics, language, habit, or even sheer luck rather than scientific achievement. Moreover, the continued use of tainted eponyms is inappropriate and will not be accepted by patients, relatives, or the public.

\section{Eponyms connected to Nazi medicine are inappropriate}

The atrocities committed by Nazi doctors are well documented ${ }^{1}$; they received new attention with the discovery that Hans Reiter, a German doctor who is remembered for his discovery of a variant of reactive arthritis, took part in human experiments. ${ }^{2}$ These revelations resulted in a decline in use of the term Reiter's syndrome, ${ }^{3}$ and a formal retraction of the eponym was proposed. ${ }^{4}$ In hindsight, the facts about Reiter escaped the scientific community only because no one had investigated the person behind the eponym.

We recently investigated the life of Friedrich Wegener, the pathologist for whom Wegener's granulomatosis is named. We discovered that Wegener had been an

\section{Eponyms lack accuracy, lead to confusion, and hamper scientific discussion}

of North America stated: "As patients and family members, we would prefer a different name for our disease," (unpublished letter to Lancet 2006). This letter and other supporting feedback to our articles on Wegener give us further confidence in our interpretation that eponyms with a connection to Nazi atrocities must be abandoned. ${ }^{8}$ Furthermore, we have been told that as a result of our research, the American College of Chest Physicians has decided to rescind its "master clinician" award to Dr Wegener.

Eponyms do not reflect scientific discoveries Use of eponyms without reflection or even knowledge of the person concerned causes other problems. A common flaw is that eponyms usually refer to one person whereas scientific discoveries often reflect a group effort over time. Behçet's disease serves as an example ${ }^{9}$ : Hulushi Behçet recognised the disease in 1937, but Benedictos Adamantiades described a case of the disease in 1930. And what about all the other people who contributed? To acknowledge everyone who discovered facets of the disorder, we would have to name it Hippocrates-JaninNeumann-Reis-Bluthe-Gilbert-PlannerRemenovsky-Weve-Shigeta-PilsGrütz-Carol-Ruys-Samek-Fischer-WalterRoman-Kumer-Adamantiades-DascalopoulosMatras-Whitwell-Nishimura-BlobnerWeekers-Reginster-Knapp-Behçet's disease. ${ }^{9}$

Similarly, Friedrich Wegener's university roommate, Heinz Klinger, described a case of what became known as Wegener's granulomatosis before Wegener. Makito Takayasu failed to recognise the vascular changes when he described the vasculitis that now bears his name. We are forced to conclude that eponymous distinction has often early member of the Nazi brown shirts and that he had been the pupil of a prolific expert on "racial hygiene." Wegener was also wanted as a war criminal, although the reasons remain unclear. ${ }^{56}$ An editorialist regarded the evidence for war crimes as "thin but tangible" and raised further questions: How heinous must an individual's behaviour be before he or she is denied eponymous distinction? ${ }^{7}$

Prompted by our revelations about Friedrich Wegener, the Vasculitis Foundation been awarded to those who had published in a more accessible journal or language and that influence, politics, or even luck have also had a role. ${ }^{10}$

\section{Eponyms lack scientific accuracy}

Eponyms are often claimed to facilitate learning and provide shorthand reference. Contrary to this intention, signs and symptoms in aortic regurgitation carry as many as 31 eponyms. ${ }^{11}$ Not surprisingly, some may remember the eponym while being unable to describe its meaning. In a systematic study, only 10 of 92 orthopaedic surgeons were able to give the correct description of Finkelstein's test for diagnosing tendovaginitis. ${ }^{12}$ Experienced trauma surgeons may spend some time in debating whether a fracture is a Barton's, a Smith II, or a reversed Barton's. ${ }^{13}$

To make matters more complicated, some diseases have different eponyms in different countries. ${ }^{10}$ For example, giant cell arteritis is known as Morbus Horton in Germany and maladie de Horton in France, but the term Horton's disease is virtually unknown in the United States. Different ways of spelling add just another level of complexity. Should ankylosing spondylitis be knows as Bechterew's disease as in Germany or Bekhterew's as in other countries? ${ }^{10} \mathrm{Or}$ Marie Strümpell disease as elsewhere in the world? Finally, eponyms may have two completely different diseases attached to them: de Quervain's disease can be tendovaginitis of the hand or a rare thyroid disorder.

Amid such confusion, it is not surprising that pleas for a more descriptive classification have emerged, particularly from specialties where eponyms were heavily used. Trauma surgeons have introduced the $\mathrm{AO}$ nomenclature, ${ }^{13}$ while neuroscientists have called for a nomenclature that refers to the site of the lesion. ${ }^{14}$

Eponyms lack accuracy, lead to confusion, and hamper scientific discussion in a globalised world. Instead of using eponyms, we should use our interest in medical history to provide fair and truthful accounts of scientific discoveries and to dissect individual contributions. We call on the editors of medical journals and textbooks to abandon the use of eponyms.

Competing interests: None declared. 
